\title{
Sistema web para la evaluación de los Programas Institucionales de Tutoría de las Instituciones de Educación Superior de la RCO de la ANUIES
}

\section{Web system for the evaluation of the Institutional Tutoring Programs of the Higher Education Institutions of the RCO of the ANUIES}

\author{
MACÍAS-BRAMBILA, Hassem Rubén ${ }^{1}{ }^{*} *$ PULIDO-GONZÁLEZ, Héctor ${ }^{2}$, BAÑUELOS- \\ MEZQUITAN, Rocío Alejandra ${ }^{2}$ y MENDOZA-MENDOZA, Luis Gerardo ${ }^{3}$
}

\author{
${ }^{1}$ Universidad de Guadalajara - Sistema de Universidad Virtual, Av. Enrique Díaz de León No. 782, Colonia Moderna, C.P. \\ 44190, Guadalajara, Jalisco, México. \\ ${ }^{2}$ Universidad de Guadalajara - Centro Universitario de Ciencias Exactas e Ingenierías, Blvd. Gral. Marcelino García \\ Barragán No. 1421, Colonia Olímpica, C.P. 44430, Guadalajara, Jalisco, México. \\ ${ }^{3}$ Universidad Tecnológica de Jalisco - División de Mecatrónica, Luis J Jiménez No. 577, Colonia Primero de Mayo, C.P. \\ 44979, Guadalajara, Jalisco, México
}

ID 1 ${ }^{\mathrm{er}}$ Autor: Hassem Rubén, Macías-Brambila / ORC ID: 0000-0002-6540-7464, CVU CONACYT ID: 902812

ID $1^{\text {er }}$ Coautor: Héctor, Pulido-González/ ORC ID: 0000-0002-8619-3012, CVU CONACYT ID: 313575

ID $2^{\text {do }}$ Coautor: Rocío Alejandra, Bañuelos-Mezquitan / ORC ID: 0000-0002-4292-1823, CVU CONACYT ID: 1067719

ID $3^{\text {er }}$ Coautor: Luis Gerardo, Mendoza-Mendoza / ORC ID: 0000-0001-8474-547X, CVU CONACYT ID: 639088

DOI: $10.35429 /$ JTAE.2020.12.4.1.9

Recibido: 24 de Junio 2020; Aceptado: 30 de Octubre, 2020

\section{Resumen}

El presente artículo describe el proceso de análisis, diseño e implementación de un sistema web para la gestión de los procesos de evaluación de los Programas Institucionales de Tutoría de las Instituciones de Educación Superior que integran la Región Centro Occidente de la Asociación Nacional de Universidades e Instituciones de Educación Superior a través de la colaboración de la Red Regional de Tutorías y el Departamento de Tutorías de la Universidad Tecnológica de Jalisco. Este proceso se desarrolló a través de la metodología ágil SCRUM por la periodicidad de las fases y su implementación. El objetivo del sistema es automatizar el proceso de evaluación de pares en el que participan los integrantes de la Red como estrategia de mejora continua y que incide directamente en la formación integral de los estudiantes, además de ser congruente con los Sistemas de Gestión de Calidad de las IES y con las recomendaciones de los Organismos Acreditadores del Consejo para la Acreditación de la Educación Superior y de los criterios de la Federación de Instituciones Mexicanas Particulares de Educación Superior como procesos que aseguran la calidad en los Programas Educativos, contribuyendo en la mejora continua de los servicios de apoyo para los estudiantes.

Sistema Institucional de Tutorías, Programa Institucional de Tutorías, Evaluación Tutorías

\begin{abstract}
This article describes the process of analysis, design and implementation of a web system for the management of the evaluation processes of the Institutional Tutoring Programs of the Higher Education Institutions that make up the Central West Region of the Asociación Nacional de Universidades e Instituciones de Educación through the collaboration of the Tutoring Network and the Tutoring Department of the Universidad Tecnológica de Jalisco. This process was developed through the agile SCRUM methodology due to the periodicity of the phases and their implementation. The objective of the system is to automate the peer evaluation process in which the members of the Network participate as a continuous improvement strategy and that directly affects the comprehensive training of students, in addition to being consistent with the Quality Management Systems of the HEIs and with the recommendations of the Accrediting Experts of the Consejo para la Acreditación de la Educación Superior and the criteria of the Federación de Instituciones Mexicanas Particulares de Educación Superior as processes that ensure quality in Educational Programs, contributing to the continuous improvement of support services for students.
\end{abstract}

Institutional Tutoring System, Institutional Tutoring Program, Tutoring Evaluation

Citación: MACÍAS-BRAMBILA, Hassem Rubén, PULIDO-GONZÁLEZ, Héctor, BAÑUELOS-MEZQUITAN, Rocío Alejandra y MENDOZA-MENDOZA, Luis Gerardo. Sistema web para la evaluación de los Programas Institucionales de Tutoría de las Instituciones de Educación Superior de la RCO de la ANUIES. Revista de Tecnología y Educación. 2020. 4-12: 1-9

\footnotetext{
* Correspondencia del Auto (Correo electrónico: hassem.macias@ academico.udg.mx)

$\dagger$ Investigador contribuyendo como primer autor.
} 


\section{Introducción}

La formación integral de los estudiantes es el elemento central que rige los modelos educativos y académicos de las Universidades e Instituciones de Educación Superior en México, cada una de ellas con diversos enfoques $u$ orientaciones humánisticas, enfocadas al emprendurismo, responsabilidad social, los valores y la relaciones interpersonales, entre otros; en este sentido la Coordinación General de Universidades Tecnológicas y Politénicas (CGUTyP) desde el Modelo Nacional de Tutorías (2017) la define como el desarrollo armónico y coherente de las dimensiones del ser humano para la realización plena del individuo en la sociedad.

En este enfoque de formación para la vida, la Asociación Nacional de Universidades e Instituciones de Educación Superior (ANUIES) desde la Región Centro Occidente (RCO) conformó la Red Regional de Tutorías desde el año 2000, iniciativa en la que diversos cuerpos colegiados de Instituciones de Educación Superior (IES) promueven el intercambio de programas, experiencias y recursos, que permiten la consolidación y el fortalecimiento de los Programas Institucionales de Tutoría (PIT) y la mejora continua, además del diseño de estrategias centradas en la acción tutorial.

La Red Regional de Tutorías de la RCO de la ANUIES está integrada por mas de 30 IES públicas y privadas, que pertencen a diversos modelos y subsistemas; y que brindan representatividad, presencia e impacto en los estados de Jalisco, Michoacán, Guanajuato, Nayarit, Aguascalientes y Colima.

En el año 2018 en la Red Regional de Tutorías se creó una comisión con las representaciones de Universidades Públicas Autonómas, Universidades Privadas, Universidades Tecnológicas e Institutos Tecnológicos, desde la cual se desarrolló la propuesta del modelo metodológico para la validación de pares de los procesos de la acción tutorial, el cual contempló los elementos básicos de modelos de excelencia y los principios de gestión de la calidad basados en la norma ISO 9000: 2015, integrando fuentes de entradas, actividades, salidas, receptores de salida e indicadores de gestión enfocados a la eficiencia, eficacia y efectividad.
Este modelo está integrado por siete ejes: fundamentos y condiciones de operación, gobierno y liderazgo, planificación, soporte, operación, evaluación del desempeño y mejora continua.

La implementación de este modelo dentro de cada una de las IES en el proceso de valoración del PIT contempla diversas fases, desde la planeación de la visita, la integración del cuerpo de evaluadores, el proceso reflexivo de autoevaluación de acuerdo a los ejes, brindando evidencia de cada uno de los criterios; así como el levantamiento de hallazgos, la generación de constancias y la presentación de las acciones de mejora encontradas en el proceso de evaluación.

La necesidad de contar con herramientas e instrumentos que faciliten el proceso de registro, seguimiento y evaluación de los criterios establecidos en los Marcos Normativos de los Organismos Acreditadores de los cuales la valoración de los PIT es uno de ellos y que además forma parte de la mejora continua de las acciones tutoriales de las IES, pretende que a través del desarrollo de un sistema web permita planear, ejecutar, documentar e informar las fases que integran el modelo metodólogico, y así valorar los elementos que conforman la acción tutorial en cada IES para incidir positivamente en la formación integral del estudiante.

\section{Metodología}

El desarrollo tecnológico se llevó a cabo durante un año, comprendido en dos periodos semestrales entre el año 2019 y 2020. Este proyecto inició con la determinación de la metodología de desarrollo de software SCRUM derivado de las características de alta adaptabilidad a los cambios de requerimientos, supuestos y dependencias, además de que brinda el marco de referencia para el desarrollo de proyectos complejos.

Esta metodología cuenta con una base fundamental, la cual se hacen entregas fragmentadas o parciales de forma continua, y además permite que el proceso de producción se genere con mayor rapidez. La metodología SCRUM contempla tres perfiles o roles que definen las responsabilidades de los equipos de trabajo en el Proyecto. 
El Project Owner (Propietario del Proyecto) el cual establece, define y orienta el objetivo general y los especifícos del proyecto, además de generar las acciones de control y verificación de las actividades del proyecto, el Master SCRUM (Experto en SCRUM) el cual se orienta principalmente en la previsión, control y resolución de los riesgos y problemas que surgen durante el desarrollo del proyecto con el equipo de trabajo, el SCRUM Team (Equipo SCRUM) integrado por el personal involucrado en el desarrollo del proyecto con roles específicos. La metodología SCRUM de acuerdo a Dimes (2015) propone el desarrollo del trabajo en cortos ciclos iterativos, denominados sprint (iteración), los que derivan en un producto o funcionalidad para entregar, lo cual convierte a esta metodología en la ideal para el desarrollo de proyectos incrementales en escenarios donde los requerimientos pueden modificarse frecuentemente, en esta implementación se trabajó en las fases de análisis, diseño, desarrollo y pruebas.

\section{Análisis}

Esta es la fase inicial del proyecto y su primer producto consiste en la integración del "Product Backlog" (Pila de producto), documento que contiene la información encontrada en la recolección de datos, después de ser análizada, categorizada y que permite caracterizar las necesidades del desarrollo que derivan en los requerimientos que deben cumplir las funcionalidades y las tareas que se realizarán.

La recolección de datos se llevó a cabo a través de entrevistas con el Departamento de Administración y Sistemas, el Departamento de Tutorías y la Representación de los Comités de Acreditación de los Programas Educativos, la Representación de las Direcciones de Carrera y la Dirección de Calidad y Proyectos Especiales de la Universidad Tecnológica de Jalisco, derivado de su experiencia en los procesos de acreditación definieron los flujos de tareas y actividades que se llevan a cabo en un proceso de evaluación a un programa, basados en el modelo metodológico desarrollado por la Red de Tutorías. A continuación se muestra el flujo del proceso de valoración:

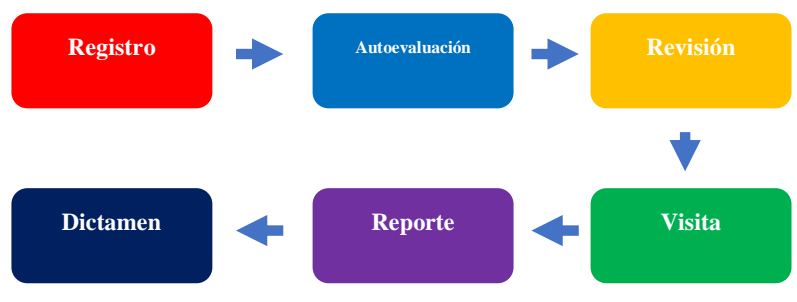

Figura 1 Flujo del proceso

Fuente: Elaboración Propia

A partir de lo anterior se determinaron las funcionalidades de un sistema a la medida que es capaz de gestionar eficientemente los procesos de planeación, documentación, seguimiento y consulta en la valoración de los PIT de las IES. El análisis de los datos recolectados y su categorización, permitió caracterizar las funcionalidades del desarrollo, iniciando con el desarrrollo del documento de Especificación de Requerimientos de Software (ERS) para lo que se utilizó la plantilla del estandar IEEE Std 8301998 del Institute of Electrical and Electronics Engineers (IEEE,1998), en este documento se define la perspectiva del producto y su funcionalidad, así como las características de los usuarios, las restricciones, suposiciones y dependencias, la evolución previsible del sistema, además de los requisitos específicos y los de interfaces: usuario, hardware, software y de comunicación, los requerimientos funcionales y no fucionales. A continuación, se muestra la descripción de algunos requerimientos específicos del ERS:

\begin{tabular}{|c|c|}
\hline $\begin{array}{l}\text { Número de } \\
\text { requisito }\end{array}$ & R1.01 \\
\hline $\begin{array}{l}\text { Nombre de } \\
\text { requisito }\end{array}$ & Crear registro de IES \\
\hline Tipo & $\triangle$ Requisito $\square$ Restricción \\
\hline $\begin{array}{ll}\text { Fuente del } \\
\text { requisito }\end{array}$ & Entrevista $12 / 09 / 2019$, registro 02 \\
\hline $\begin{array}{l}\text { Prioridad del } \\
\text { requisito }\end{array}$ & \begin{tabular}{|ll} 
Alta/Esencial & $\square$ \\
Media/Deseado
\end{tabular} \\
\hline
\end{tabular}

\begin{tabular}{|ll||ll|}
\hline $\begin{array}{l}\text { Número } \\
\text { requisito }\end{array}$ & de & R1.07 \\
\hline $\begin{array}{l}\text { Nombre } \\
\text { requisito }\end{array}$ & de & Personalizar imagen institucional de la IES \\
\hline Tipo & & Q Requisito & $\square$ Restricción \\
\hline $\begin{array}{l}\text { Fuente } \\
\text { requisito }\end{array}$ & del & Entrevista 12/09/2019, registro 04 & \\
\hline $\begin{array}{l}\text { Prioridad } \\
\text { requisito del }\end{array}$ & X & \\
\hline
\end{tabular}

\begin{tabular}{|c|c|}
\hline \begin{tabular}{ll||} 
Número & de \\
requisito &
\end{tabular} & R3.02 \\
\hline $\begin{array}{l}\text { Nombre de } \\
\text { requisito }\end{array}$ & Crear cuentas de usuario y asignar perfil \\
\hline Tipo & $\triangle$ Requisito $\square$ Restricción \\
\hline \begin{tabular}{ll|}
$\begin{array}{l}\text { Fuente } \\
\text { requisito }\end{array}$ & del \\
\end{tabular} & Entrevista 12/09/2019, registro 07 \\
\hline $\begin{array}{l}\text { Prioridad del } \\
\text { requisito }\end{array}$ & \begin{tabular}{|ll} 
冈lta/Esencial & $\square$ Media/Deseado
\end{tabular} \\
\hline
\end{tabular}




\begin{tabular}{|lr||ll|}
\hline $\begin{array}{l}\text { Número } \\
\text { requisito }\end{array}$ & de & R4.20 \\
\hline $\begin{array}{l}\text { Nombre } \\
\text { requisito }\end{array}$ & de & Exportar plan dictamen \\
\hline Tipo & & Q Requisito $\quad \square$ Restricción & \\
\hline $\begin{array}{l}\text { Fuente } \\
\text { requisito }\end{array}$ & del & Entrevista 10/10/2019, registro 12 & \\
\hline $\begin{array}{l}\text { Prioridad } \\
\text { requisito del }\end{array}$ & D & \\
\hline
\end{tabular}

\begin{tabular}{|ll||ll|}
\hline $\begin{array}{l}\text { Número } \\
\text { requisito }\end{array}$ & de & R5.17 \\
\hline $\begin{array}{l}\text { Nombre } \\
\text { requisito }\end{array}$ & de & Exportar Constancias de participación \\
\hline Tipo & & Q Requisito $\quad \square$ Restricción & \\
\hline $\begin{array}{l}\text { Fuente } \\
\text { requisito }\end{array}$ & del & Entrevista 10/10/2019, registro 15 & \\
\hline $\begin{array}{l}\text { Prioridad } \\
\text { requisito del }\end{array}$ & X & \\
\hline
\end{tabular}

Tabla 1 Especificación de requerimientos Fuente: Elaboración Propia

Después de la definición del ERS, se construyó la Sprint Backlog (lista de tareas), en este documento de delimitan las tareas y acciones que se deben llevar a cabo, además de asignar a las personas del SCRUM Team que serán las responsables de ejecutarlas; en esta fase es necesario determinar el periodo en que deben llevarse a cabo estas tareas, para lo que se realizó un Diagrama de Gantt y un Gráfico de Pert, para controlar la programación de las tareas.

La determinación de los Sprint permite realizar pruebas en un modelo incremental y además la ejecución de Daily SCRUM (reunión diaria), donde se incluye la discusión de todo lo desarrollado, lo que se planea implementar y la gestión de riesgos detectados en el proceso de desarrollo.

\section{Diseño}

Esta fase contempla el diseño arquitectónico del sistema y el semántico de los metadatos; el modelado del sistema incluyó el desarrollo del diagrama de bloques identificando las entradas y salidas del sistema, así como los bloques con las funcionalidades principales y la interacción que existirá entre cada uno de los módulos. A continuación, se muestra un extracto del diagrama de bloques:

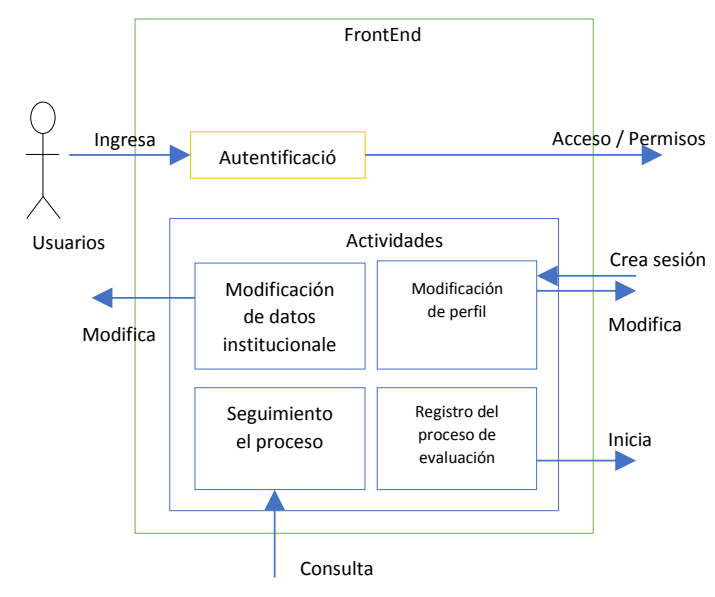

Figura 2 Extracto del diagrama de bloques Fuente: Elaboración Propia

La determinación de los módulos del sistema facilitó el desarrollo de los modelos que propone el Lenguaje Unificado de Modelado (UML) para el diseño estático y dinámico de los componentes de software, siendo el diagrama de clases el primero que se diseñó identificando a través de esté las clases que componen el software, sus atributos, métodos y las relaciones que deben establecerse.

De la misma manera; incluyendo buenas prácticas el modelado de sistemas, y con el objetivo de ofrecer un mayor detalle en la viabilidad de los componentes y sus relaciones, se diseñaron las tarjetas Clase-ResponsabilidadRelación (CRC). A continuación, se muestra la tarjeta CRC de categoría:

\begin{tabular}{|l|l|}
\hline \multicolumn{2}{|c|}{ Indicador } \\
\hline Superclase: categoría & Métodos \\
\hline Atributos & $\begin{array}{l}\text { + Vinculacion(): void } \\
+ \text { setNombre(varchar[]): void } \\
+ \text { getNombre(): varchar[] } \\
+ \text { setCategoria(varchar[]): void } \\
+ \text { getCategoria(): varchar[] }\end{array}$ \\
\hline $\begin{array}{l}\text {-codigo: integer (4) } \\
\text { - nombre: varchar(40) }\end{array}$ & Colabororaciones \\
\hline Reschar(40) & Categorías \\
\hline Vinculación de categorías & Usuario \\
\hline $\begin{array}{l}\text { Clasificación de respuestas por tipo } \\
\text { de usuario }\end{array}$ & NA \\
\hline $\begin{array}{l}\text { Clasificación de respuestas por } \\
\text { indicador }\end{array}$ & \\
\hline
\end{tabular}

Tabla 2 Tarjeta CRC categoría

Fuente: Elaboración Propia

Otro elemento de diseño fue el diagrama de objetos, el cual permitió visualizar la comunicación, relación, entradas y salidas entre las instancias que compartiran relacionesmensajes de acuerdo a la estrucutura estática de las clases. 
Derivado del proceso anterior se desarrollaron el diseño y el desarrollo de los casos de uso, desde el modelado gráfico rescatando las interacciones y detonantes mediante actores, hasta la definición de los procesos, su vínculo con las entidades del sistema y las precondiciones y postcondiciones, así como los supuestos y las restricciones de cada una de las funcionalidades. A continuación, se muestra el caso de uso de administración de evaluación:

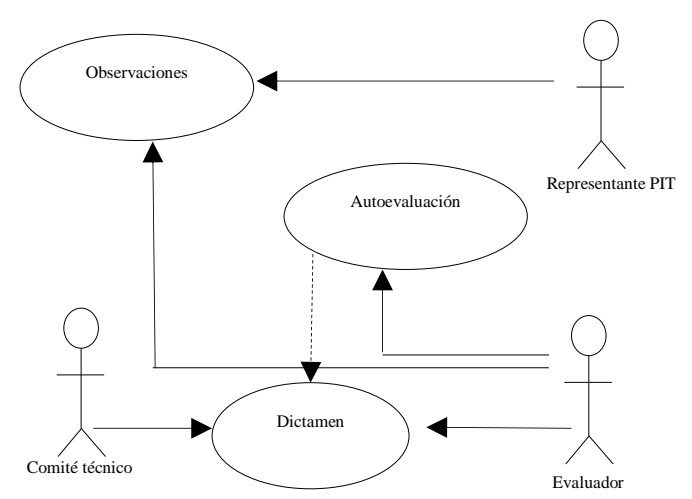

Figura 3 Diagrama de casos de uso administración evaluación

Fuente: Elaboración Propia

El modelado de los casos de uso permitió su definición, y sobre todo la especificación de cada uno de los procesos. A continuación, se muestra la definición del caso de uso agregar evaluador:

\begin{tabular}{|c|c|c|c|}
\hline \# & $\begin{array}{l}\text { Actividad } \\
\text { de actor }\end{array}$ & $\begin{array}{l}\text { Actividad de sistema } \\
\text { [Descripción/Cálculo] }\end{array}$ & $\begin{array}{l}\text { [Condición] } \\
\text { Alternativa }\end{array}$ \\
\hline 1 & $\begin{array}{l}\text { Presionar } \\
\text { el botón } \\
\text { de agregar } \\
\text { un } \\
\text { evaluador }\end{array}$ & $\begin{array}{l}\text { El sistema despliega la } \\
\text { vista de la creación de } \\
\text { usuarios, con todos los } \\
\text { datos a llenar }\end{array}$ & $\begin{array}{l}\text { - Si la conexión a } \\
\text { internet falla, la } \\
\text { vista no puede } \\
\text { cargarse }\end{array}$ \\
\hline 2 & $\begin{array}{l}\text { Ingresar } \\
\text { datos }\end{array}$ & $\begin{array}{l}\text { El sistema validará la } \\
\text { información y la } \\
\text { almacenará en la base } \\
\text { de datos }\end{array}$ & $\begin{array}{l}\text { - } \mathrm{Si} \text { no existen } \\
\text { evaluaciones } \\
\text { registradas } \\
\text { aparecerá en blanco } \\
\text { el combo box } \\
\text { - Si la conexión con la } \\
\text { base de datos falla, } \\
\text { el sistema no } \\
\text { almacenará la } \\
\text { información } \\
\text { - Si un campo queda } \\
\text { vacío no se podrá } \\
\text { guardar }\end{array}$ \\
\hline 3 & & $\begin{array}{l}\text { El sistema guardará un } \\
\text { registro en otra tabla } \\
\text { con el código del } \\
\text { evaluador } \\
\text { evaluación. }\end{array}$ & \\
\hline
\end{tabular}

Tabla 3 Definición de caso de uso agregar evaluador

Fuente: Elaboración Propia
La definición de los casos de uso derivó al proceso de identificación de actores, lo que posibilitó la definición de las entradas y salidas desde cada uno de los detonantes de los casos de uso, al igual que la definición de entidades en el cual se determinaron las acciones CRUD (create, read, update y delete) de los registros de cada una de las entidades con respecto al caso de uso que las afecta.

El diseño semántico de datos se incluyó la definición de los metadatos que se implementarían en el sistema, iniciando con un diseño conceptual del proceso a través del diagrama Entidad-Relación (DER). A continuación, se muestra un extracto del DER:

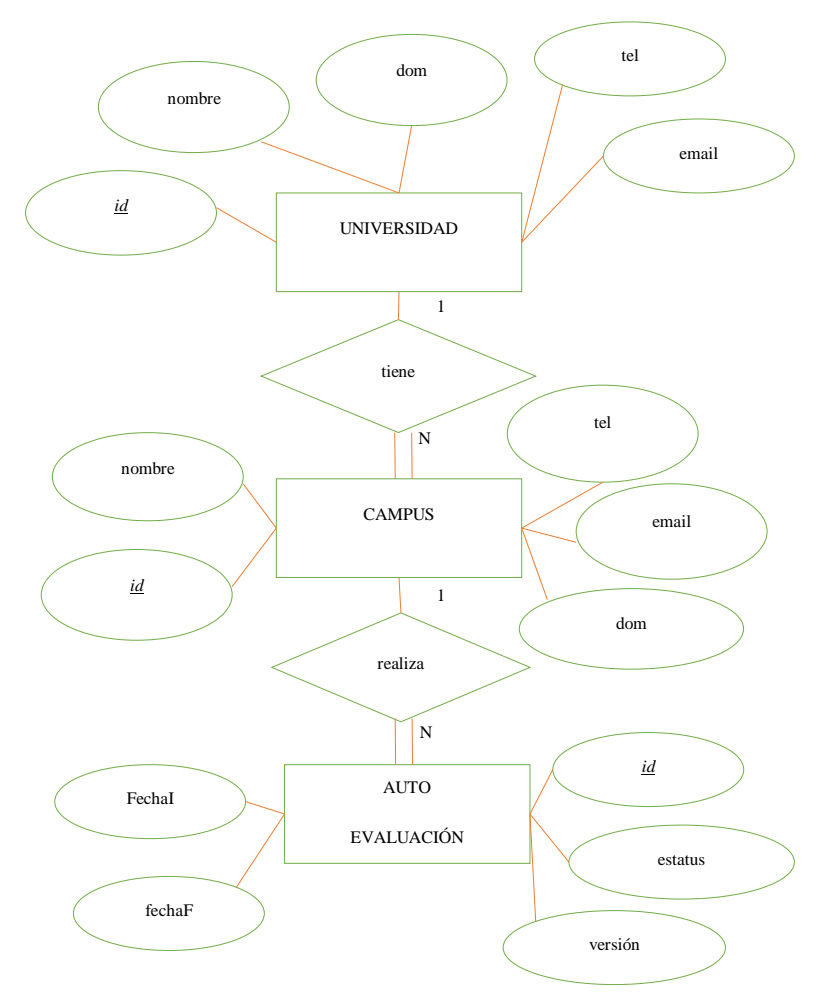

Figura 4 Extracto del DER

Fuente: Elaboración Propia

La normalización de la base de datos y la verificación de las restricciones funcionales, permitió la construcción del modelo de implementación, a través del modelo relacional, del cual se muestra un extracto a continuación: 


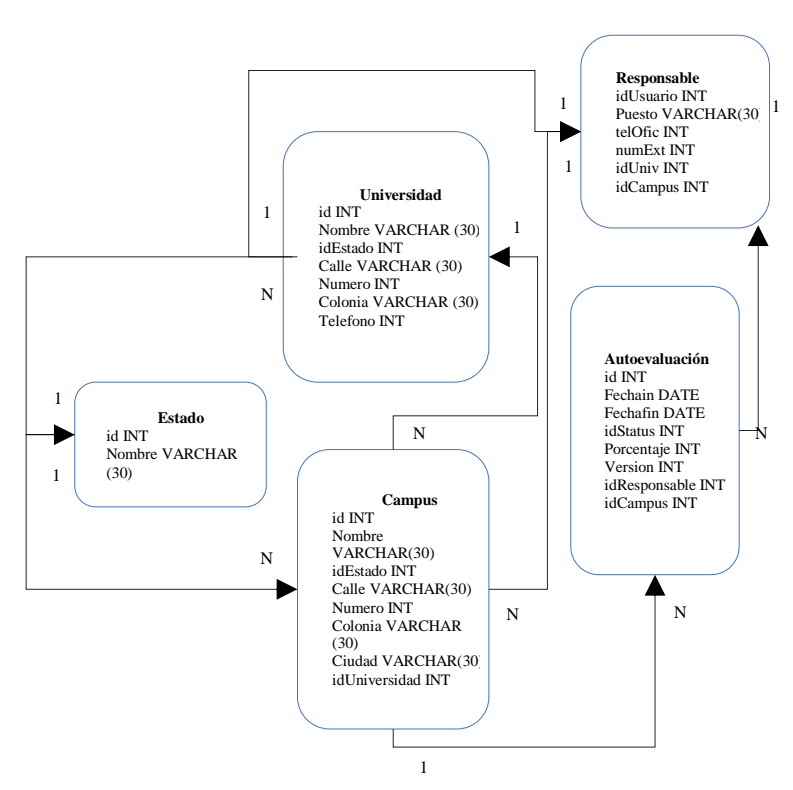

Figura 5 Extracto del modelo relacional Fuente: Elaboración Propia

\section{Desarrollo}

La fase de producción del software se llevó a cabo con la colaboración y dirección de proyectos modulares en los que participaron estudiantes del PE de Ingeniería en Computación del Centro Universitario de Ciencias Exactas e Ingenierías de la Universidad de Guadalajara, en donde se utilizó Sublime Text como editor de texto y código fuente.

Este proceso incluyó la implementación del diseño a través del patrón Modelo-VistaControlador (MVC), favoreciendo la administración entre las interacciones del usuario y los componentes del sistema. A continuación, se muestra la interfaz del menú de información del usuario:

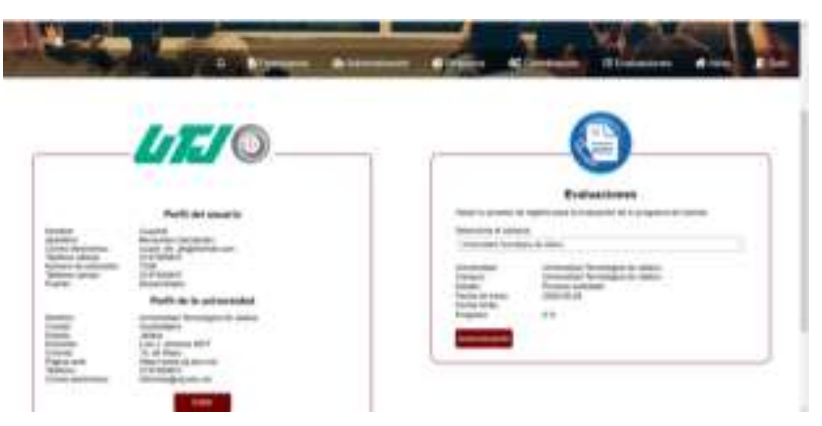

Figura 6 Interfaz de información de usuario Fuente: Elaboración Propia

El sistema está diseñado para realizar ajustes en los procesos de evaluación, tales como las actualizaciones a los marcos normativos, guías o criterios de evaluación. A continuación, se muestra la interfaz de ajustes de autoevaluación:

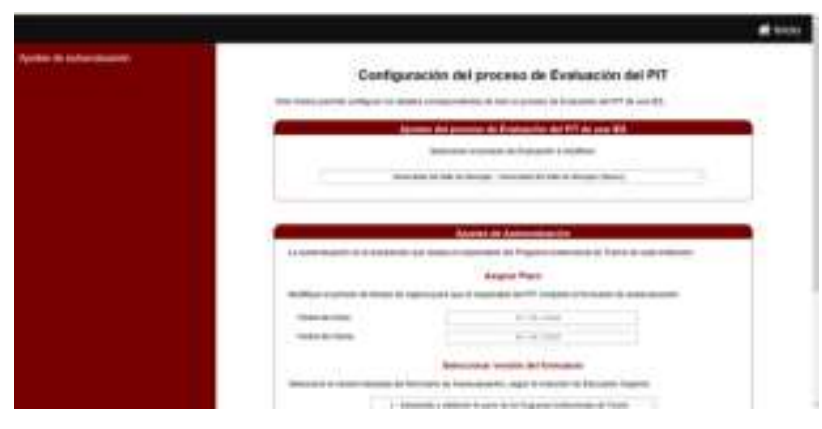

Figura 7 Interfaz de ajustes de autoevaluación Fuente: Elaboración Propia

La adaptabilidad del sistema es un elemento fundamental ya que contempla la personalización para los procesos de gestión de instituciones, donde tienen incluso varios campus o unidades académicas y los procesos de evaluación pueden distinguirse entre cada una de las sedes de una Universidad. A continuación, se muestra la interfaz de campus:

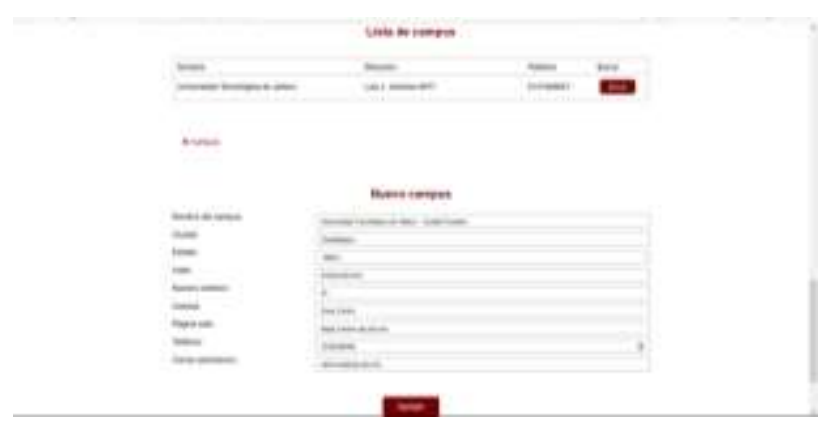

Figura 8 Interfaz de gestión de campus Fuente: Elaboración Propia

El seguimiento en el avance de cada una de las fases del proceso de evaluación permite que los actores de los comités involucrados y los representantes de las IES estén informados del progreso en el ejercicio. A continuación, se muestra la interfaz de notificaciones:

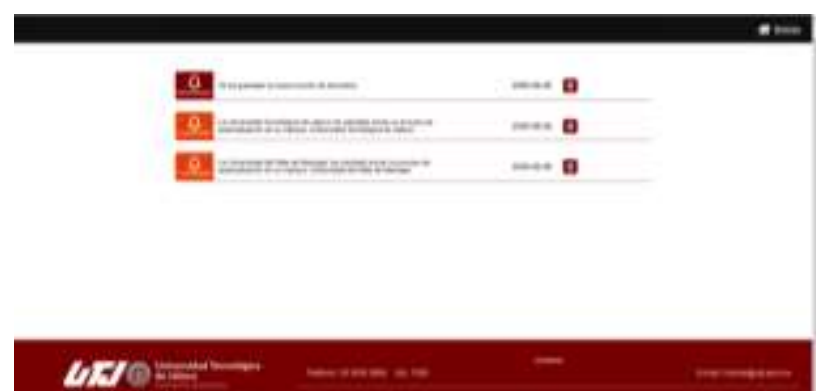

Figura 9 Interfaz de notificaciones

Fuente: Elaboración Propia

MACÍAS-BRAMBILA, Hassem Rubén, PULIDO-GONZÁLEZ, Héctor, BAÑUELOS-MEZQUITAN, Rocío Alejandra y MENDOZA-MENDOZA, Luis Gerardo. Sistema web para la evaluación de los Programas Institucionales de Tutoría de las Instituciones de Educación Superior de la RCO de la ANUIES. Revista de Tecnología y Educación. 2020 


\section{Pruebas}

En esta fase se diseñó e implementó un plan de pruebas que contempla información técnica del proyecto, el historial de versiones y el alcance de las pruebas, en el que se especificó:

\section{- $\quad$ Elementos de pruebas \\ - Funcionalidades a probar \\ - $\quad$ Pruebas de regresión \\ - $\quad$ Funcionalidades que no serán probadas \\ - $\quad$ Estrategia de pruebas}

Además; con el objetivo de determinar un desempeño aproximado de la ejecución de los Casos de Uso, se definieron a través de los requerimientos funcionales y no funcionales, los criterios de aceptación y rechazo de las pruebas a través de un comportamiento binario. Así como la determinación de los entregables, los recursos, la planeación y la organización para la ejecución del plan de pruebas.

\section{Resultados}

Se estableció un sistema de almacenamiento de datos centralizado para la administración en la Universidad Tecnológica de Jalisco, debido a que la estimación realizada de acuerdo a la capacidad operativa de una jornada de visitas y evaluaciones en las IES no contempla una conexión simultánea mayor a 30 usuarios aproximadamente, por lo que las peticiones de los clientes, la gestión del almacenamiento de datos y del procesamiento de operaciones, no justifican el costo operativo, tecnológico y monetario de una base de datos distribuida.

La determinación del entorno de desarrollo, de instalación y configuración del servidor no se contempló ningún tipo de restricción, excepto en la adquisición de licencias, por lo que se optó por la implementación de tecnologías y herramientas CASE (Computer Aided Software Engineering) libres, lo que favorece la adaptación de las tecnologías a sus procesos al no representar costos de licenciamiento.

Se realizaron a través del plan de pruebas, casos que contemplaron aspectos de conectividad, rendimiento, interfaz y funcionalidad; para los que se comprobó efectivamente la conexión simultánea de 45 usuarios y sus respectivas peticiones como clientes, así como las salidas de los procesos.
A través de estadística descriptiva se determina la coincidencia en un $97.56 \%$ con los resultados esperados en los casos de prueba. El resto de las salidas que no cumplieron los criterios de aceptación se documentaron y se reprocesaron.

\section{Conclusiones}

La automatización y sistematización del proceso de evaluación de los PIT de las IES establece un marco operativo para la administración de los registros y evidencias que sustentan la operación, eficiencia, pertinencia y suficiencia del programa de acuerdo con las características institucionales y a la normatividad vigente para el Subsistema al que pertenece la Institución.

Este proceso sistematizado es congruente con los objetivos de calidad y ambientales de la mayoría de las Instituciones, el disminuir la utilización de medios impresos para evidenciar procesos que son evaluados, además de alinear el PIT a un proceso de mejora continua que incida en la formación integral de los estudiantes, evaluando el impacto de las acciones tutoriales en la población estudiantil, y con base a los resultados obtenidos establecer un plan de mejora continuo, que será evaluado periódicamente para su actualización y ajustes que se requieran.

Este proyecto es la primera fase en la valoración de los Servicios de Apoyo al Estudiante, por medio de la evaluación de pares que facilita la estructura de la RCO de la ANUIES a través de la red regional de Tutorías; experiencia que permitirá permear este ejercicio de evaluación sistematizado al resto de las redes regionales de las mismas instituciones, para valorar mediante un modelo metodológico y el apoyo de una herramienta tecnológica el resto de los servicios de apoyo al estudiante que influyen en su permanencia en la Institución y en su Formación Integral, ya que la inexistencia de estas herramientas se considera como uno de los elementos que limitan o dificultan los procesos de acreditación de los PE.

\section{Agradecimientos}

Agradecemos el apoyo y colaboración de los estudiantes, profesores, administrativos y directivos de las Instituciones de Educación Superior que impulsaron e hicieron posible el desarrollo de esta herramienta tecnológica. 
En especial agradecemos la colaboración del comité del modelo de valoración de PIT en la IES integrado por los representantes de la Universidad de Celaya el Dr. Juan Antonio Zacarías Sánchez, la Universidad del Valle de Atemajac la Mtra. María Cristina Dávila Avendaño, la Universidad Michoacana de San Nicolás de Hidalgo la Dra. Dra. Marcela Patricia del Toro Valencia, la Universidad Autónoma de Aguascalientes la Lic. Lic. Karina Gutiérrez Juárez de la Red Regional de Tutorías de la RCO de la ANUIES, y a todo el personal de la Universidad Tecnológica de Jalisco que lo hizo posible.

\section{Referencias}

Aguiar, B. O., Velázquez, R. M., \& Aguiar, J. L. (2019). Innovación docente y empleo de las TIC en la Educación Superior. Revista ESPACIOS, 40(02).

Albújar Sobrino, K. X., \& Torres Seminario, D. C. (2019). Análisis y diseño de software para la automatización de historias clínicas del Policlínico UDEP.

Asociación Nacional de Universidades e Instituciones de Educación Superior. (2020). Mayo 25, 2020, de ANUIES RCO Sitio web: http://www.anuiesrco.org.mx/redesregionales/red-de-tutorias

Consejo para la Acreditación de la Educación Superior, A.C. (2016) Marco General de Referencia para los Procesos de Acreditación de Programas Académicos de Tipo Superior. COPAES.

Coordinación General de Universidades Tecnológicas y Politécnicas. (2017). Modelo Nacional de Tutorías. Secretaría de Educación Pública.

Date, C.J. (2001). Introducción a los sistemas de bases de datos. Pearson Education. 251-253.

Durango, Alicia. (2014). Diseño web con CSS. Createspace Independent Pub. 11-15.

Espinoza Freire, Eudaldo E.; Ley Leyva, Nelly V. \& Guamán Gómez, Verónica J.(2019). Papel del tutor en la formación docente. Dialnet.230241 .
González Basilio, Sofía J.; López Nomezqui, Wendy S.; Rivera Delgado, Perla I.; Cárdenas Ayala, María T. \& Pérez Ocampo, Hugo F.(2019). Propuesta de tutoría virtual para los programas educativos en modalidad no escolarizada de la Universidad Autónoma de Nayarit. MICA. 97-105.

Hernández, Giovanni; Martínez, Álvaro; Jiménez, Robinson \& Jiménez, Franklin. (2019). Scrum y peopbleware: elementos clave para la gestión en la construcción de software. Risti. 265-277.

IEEE. (1998). IEEE Std 830-1998 - IEEE Recommended Practice for Software Requirements Specifications. mayo 20, 2017, de C - IEEE Computer Society Sitio web: https://standards.ieee.org/findstds/standard/8301998.html.

Lagunas, J. R., Piña, M. A. L., \& Vázquez, J. M. H. (2020). La reforma de la educación en México, entre la espada y la pared. La Mirada de los Universitarios. Revista Polis, 16(1), 7-31

Macías Brambila, Hassem R., López Laguna, Ana B., González del Castillo, Edgardo E., \& Tolosa Carrillo, Esaú. (2017). Servidor de aplicaciones como evidencia para sinergia academia-empresa MyPyMES de México. Revista de Tecnología Informática. Ecorfan. 3943.

Macías Brambila, Hassem R., López Laguna Ana B., Peña Montes de Oca, Adriana I., \& Álvarez Jiménez, Hugo R. (2017). Web Development: Evidence of follow-up for compliance with the UN Global Compact in Construction Companies. Journal-Republic of Paraguay. Ecorfan. 20-26.

Minguet, P. A., \& Solís, A. U. (2019). Educación y Sostenibilidad en la Universidad de Valencia: construyendo futuro desde el pasado. Revista de Educación Ambiental y Sostenibilidad, 12021202.

Pérez, M. M. D., \& Aguilar, B. L. C. (2020). Estudio para realizar la acción tutorial a través de un sistema de gestión de aprendizaje en Moodle para el nivel de secundaria. MLS Educational Research, 4(1). 
Roberto, P. M. E., Georgina, S. P. E., \& Félix, G. R. J. (2020). Validación de modelo de medición de satisfacción estudiantil universitaria con los servicios académicos recibidos. Investigación Operacional, 41(3), 472-483

Rojas, Esperanza M.; Ramírez, Margarita; Ramírez Moreno, Hilda. B.; Salgado Soto, María C. \& Millán Osuna, Nora C. (2019). Sistema de gestión académica a través del desarrollo de modelo-vista-controlador. Risti. 1083-1093.

Romero J. Especificación de Requisitos Software según el estándar IEEE 830. Recuperado el día 8 de agosto de 2017 de: https://www.fdi.ucm.es/profesor/gmendez/docs/ is0809/ieee830.pdf

Ruiz, I. I. B. (2019). Learning Analytics como cultura digital de las universidades: Diagnóstico de su aplicación en el sistema de educación a distancia de la UNAM basado en una escala compleja. Revista Iberoamericana de Educación, 80(1), 89-116.

Sánchez González, M. J. (2019). Desarrollo e implementación de sistema web de registro de pagos de alícuotas para automatización de control de acceso de vehículos en urbanización privada.

Dimes, Troy. (2015). Conceptos básicos de Scrum: desarrollo de software agile y manejo de proyectos agile. Babelcube. 9-14.

Sommerville, I. (2011). Software Engineering (9 ed.). Person.

Terrazas Pastor, Rafael (2011). Planificación y programación de operaciones. Scielo. 8-11.

Tuya, Javier; Ramos Roman, I.\& Dolado Cosín, J. (2007). Técnicas cuantitativas para la gestión en la ingeniería de software. Netbiblio. 49-53. 